\title{
Development of the Metronomic Biofeedback Pump for leptomeningeal carcinomatosis: technical note
}

\author{
Thomas C. Chen, MD, PhD, ${ }^{1}$ Gina R. Napolitano, BS, ${ }^{2}$ Frank Adell, BS, ${ }^{2}$ Axel H. Schönthal, PhD, ${ }^{3}$ \\ and Yehoshua Shachar, BS 2,4
}

\begin{abstract}
Departments of ${ }^{1}$ Neurosurgery and Pathology, and ${ }^{3}$ Molecular Microbiology \& Immunology, Keck School of Medicine, University of Southern California, Los Angeles; ${ }^{2}$ Pharmaco-Kinesis Corporation, Inglewood; and ${ }^{4}$ Magnetecs Corporation, Inglewood, California
\end{abstract}

\begin{abstract}
Patients with leptomeningeal carcinomatosis face a particularly grim prognosis. Current treatment consists of intrathecal delivery of methotrexate (MTX) or cytosine arabinoside (Ara-C) via Ommaya reservoir or lumbar puncture. Yet despite these interventions, the median survival after diagnosis is only 4-7 months. To address inherent shortcomings of current treatments and provide a more effective therapeutic approach, the Pharmaco-Kinesis Corporation has developed a novel type of implantable pump capable of delivering intrathecal chemotherapy (i.e., MTX) in a metronomic fashion with electronic feedback. The Metronomic Biofeedback Pump (MBP) consists of 3 components: 1) a 2-lumen catheter; 2) a microfluidic delivery pump with 2 reservoirs; and 3) a spectrophotometer monitoring MTX concentrations in the CSF. Using an animal model of intraventricular drug delivery, the authors demonstrate that the MBP can reliably deliver volumes of $500 \mu \mathrm{l} / \mathrm{min}$, consistently measure real-time intrathecal MTX concentrations via CSF aspiration, and provide biofeedback with the possibility of instant control and delivery adjustments. Therefore, this novel approach to chemotherapy minimizes toxic drug levels and ensures continuous exposure at precisely adjusted, individualized therapeutic levels. Altogether, application of the MBP is expected to increase survival of patients with leptomeningeal carcinomatosis, and appropriate Phase I and II trials are pending.
\end{abstract}

http://thejns.org/doi/abs/10.3171/2014.10.JNS14343

KEY WORDS biofeedback; intrathecal; leptomeningeal carcinomatosis; metronomic delivery; oncology

\section{$\mathrm{L}$} EPTOMENINGEAL carcinomatosis (LC) is a condition in which cells from a primary solid or hematological tumor metastasize, invade the subarachnoid space, and spread throughout the CNS via the CSF, resulting in seeding of the leptomeninges in a diffuse and multifocal manner along the surface of the brain and spinal cord. It usually represents a late event of cancer progression and is most commonly observed in patients with advanced breast and lung cancer, as well as in melanoma. The most frequent symptoms include multiple cranial nerve deficits, motor deficits, altered mental status, headache, and radicular pain..$^{3,16}$
In the US, approximately $1 \%-8 \%$ of cancer patients are diagnosed with LC, but on autopsy it is detected in $19 \%$ of those in whom disseminated disease presents with neurological signs and symptoms. The incidence of LC has been reported to be increasing, due to longer overall survival in patients treated with novel antineoplastic agents. There is currently no cure and outcome is invariably fatal. The median survival of patients with $\mathrm{LC}$ receiving current therapy is a mere 3.6 months, 4 months, and 7 months from the time of diagnosis in the cases of melanoma, small-cell lung cancer, and breast carcinoma, respectively.,17

The treatment of LC rarely involves systemic chemo-

ABBREVIATIONS Ara-C = cytosine arabinoside; BBB = blood-brain barrier; GUI = graphical user interface; ICP = intracranial pressure; LC = leptomeningeal carcinomatosis; $\mathrm{MBP}=$ Metronomic Biofeedback Pump; MTX = methotrexate.

SUBMITTED April 8, 2014. ACCEPTED October 31, 2014.

INCLUDE WHEN CITING Published online May 8, 2015; DOI: 10.3171/2014.10.JNS14343.

DISCLOSURE Dr. Chen and Mr. Shachar own US Patent \#7799016, protecting the Metronomic Biofeedback Pump (MBP) system. Both have ownership interest in Pharmaco-Kinesis; also, Mr. Adell is the CEO of Pharmaco-Kinesis. A US trademark application has been filed for the MBP and was assigned serial number 86404714. 
therapy because most agents do not penetrate the bloodbrain barrier (BBB) at concentrations that are sufficiently high to exert therapeutic activity. Instead, current options for treating LC are limited to intrathecal chemotherapy, either via repeated lumbar punctures or via a surgically placed Ommaya reservoir with intraventricular catheter. Lumbar injections are difficult for a patient to tolerate, and it is not entirely clear to what extent chemotherapy instilled into the lumbar thecal sac can spread to the brain and the cervical and thoracic spinal cord. Therefore, the currently preferred method is the Ommaya reservoir, which delivers chemotherapy directly to the ventricular system and has been shown to be more effective than the lumbar puncture. . $^{1,6,21}$

To date, only 2 chemotherapeutic agents are commonly used for delivery via the Ommaya catheter: methotrexate (MTX) or cytosine arabinoside (Ara-C), which traditionally have been given as single-dose injections 2 days per week during an induction phase, followed by less frequent injections during subsequent consolidation and maintenance phases. However, because of the short half-life and rapid clearance of these medications, more frequent dosing has been applied also, but this latter approach is more taxing on the patient. A more recent approach involves a liposomal formulation of Ara-C, called DepoCyt (Sigma-Tau Pharmaceuticals, Inc.) that provides for a longer half-life of the drug and allows for less frequent injections (once every $2-4$ weeks). ${ }^{8}$

Still, treatments via Ommaya reservoir have inherent limitations that minimize therapeutic efficacy and impair patient convenience, with inherent complications related to placement and delivery. ${ }^{1,2,6}$ Therapeutic efficacy is hampered by a lack of clear understanding of the amount of drug delivered and the steady-state levels achieved in the CSF. Moreover, despite individualizing the dosage based on patient body-size measurements, the toxicity and efficacy outcomes of chemotherapy vary considerably among patients. This occurs due to the highly variable interindividual pharmacokinetics of anticancer agents, as well as unpredictable changes in CSF flow dynamics that may interfere with and disturb therapeutic levels of drug exposure. ${ }^{5,7}$ Moreover, bolus injections are not conducive to maintaining drug dosages at fine-tuned therapeutic levels for extended time periods. ${ }^{5}$ Patient convenience and quality of life are impaired by the need for the patient to come to the outpatient hospital for injections, with a significant time commitment for both patient and doctor-and in fact, the popularity of DepoCyt over MTX has not been so much a function of DepoCyt's efficacy, but of its convenience. Finally, puncturing the skin to access the Ommaya reservoir increases the risk of infections. ${ }^{12}$

To address these issues, the Pharmaco-Kinesis Corporation has developed a novel type of implantable infusion pump with several highly attractive features aimed at overcoming limitations of currently used intrathecal delivery procedures. The Metronomic Biofeedback Pump (MBP) supports the following functions: 1) controlled metronomic drug delivery over extended time periods; 2) real-time measurements of drug concentrations in the CSF; and 3) instant adjustments of the rate of drug delivery based on continuous system biofeedback. Therefore, clinical appli- cation of the MBP is expected to avoid detrimental (and currently undetectable) fluctuations of drug concentrations in the CSF and allow highly individualized, continuous exposure at precisely maintained therapeutic levels. Moreover, it allows the physician to have full control of the device, with options to modify the treatment regimen promptly according to changing conditions.

In this report, we demonstrate the capabilities of the MBP with in vitro and in vivo experiments that simulate and accomplish intraventricular delivery of MTX. The results from the in vitro experiments are compared with a computer simulation model to visualize the accuracy of the delivered dosages. Our in vivo experiments in swine present the use of a spectrophotometer as a real-time feedback sensor to monitor MTX levels in CSF, enabling pharmacokinetically guided delivery of chemotherapeutic agents with the goal of maximizing therapeutic outcomes.

\section{Methods \\ The MBP Implantable Infusion System}

The MBP is part of a multicomponent system that includes the following subsystems. 1) The MBP itself, which is an implantable infusion pump with sampling and diagnostic capabilities. 2) A 2-lumen catheter reservoir arrangement that enables both local delivery of medications and the sampling of biological fluids from the delivery site. (Alternatively, both lumens can be used to deliver 2 different therapeutic agents for combination therapy purposes.) 3) An external wireless transmitter and receiver with programmer and data collection system (i.e., a graphical user interface [GUI]) that communicates with the MBP via remote wireless connection, and is used to program and display sensor data from the MBP.

\section{The MBP Subsystem}

The enclosure of the MBP is made of a medical-grade, lightweight titanium alloy that is widely used in many longterm implant devices and enables MRI compatibility. Two micropumps regulated by valves, whose flow rates can be calibrated to approximately $500 \mu \mathrm{l} / \mathrm{min}$, enable simultaneous pumping of drugs from reservoirs to the brain, and acquire CSF samples for analysis by the onboard sensor. The MBP contains two 5-ml reservoirs, which can be configured for standard metronomic and biofeedback applications or for polypharmacy use. The standard metronomic and biofeedback option has one reservoir for drug delivery and another for storing sample fluid. The polypharmacy application has each reservoir containing a different drug, which can be selected on demand by clinicians. The microfluidics card allows for delivery of medication from the chemotherapy reservoir, or to transport CSF for sampling. A 4-wavelength spectrophotometer $(355,365,395$, and $470 \mathrm{~nm}$ ) is used to determine concentrations of MTX in CSF in real time, based on the characteristic ability of MTX to absorb ultraviolet light at $370 \mathrm{~nm} .{ }^{20}$ The 4-wavelength spectrophotometer has been tested and is shown to be effective in monitoring MTX concentrations in real time. This will measure the distribution and elimination of drugs, and enable physiological feedback on treatment response. Sensors allow for autonomous operation and 
improve device safety. The MBP is equipped with pressure sensors to measure reservoir volume, detect channel blockages, and monitor intracranial pressure (ICP). A flow-rate sensor controls the drug delivery rate, and biosensors detect medication concentration. A thermistor is included to monitor temperature fluctuations.

\section{Specialized Catheter System}

The MBP uses a specialized 2-lumen multipiece catheter. This subcutaneous catheter connects the MBP to a delivery location such as a lateral ventricle for the treatment of LC. The 2 noncommunicating lumens are used as follows: one lumen is used to deliver medication from the pump to the treatment site, and a second lumen is used for transporting biological fluid samples from the treatment site back to the pump, where they are analyzed and contained for later removal. In clinical use, the catheter will be implanted much like a ventricular shunt catheter, the main difference being that the proximal end of the catheter will be connected to the MBP, which in turn is implanted above the pectoralis muscle.

\section{Communication Components}

The MBP is equipped with a low-power wireless radio, operating in a Federal Communications Commission-approved medical implant wireless band intended for application in implanted medical devices, such as pacemakers, implanted cardioverter defibrillators, neurostimulators, and drug pumps. It is composed of an internal wireless system and integrated antenna, as well as an external antenna for transmitting and receiving data. The MBP can be monitored and controlled by the clinician to remotely administer a highly effective therapeutic regimen, thereby allowing the physician full control of the device and the ability to update treatment regimens according to changing conditions.

\section{The MBP Programmer and Data Collection Base Station}

The MBP uses a medical-grade wireless chip and protocol, which enables it to transfer and receive data and commands after implantation. To schedule the delivery of drugs, as well as the sampling of biological fluid from the treatment site, a wireless base station is used. This base station communicates with the MBP via a connected wireless antenna. The base station runs a GUI from which schedules can be set and reviewed for CSF sampling and drug delivery. Sensor data can also be viewed in near real time, and schedules for periodic sensor data collection can be set. In addition, the GUI allows the user to view the status of the device remotely. Items such as battery life, medication level, catheter clogging, and patient activity can be monitored through the base station.

\section{In Vitro Simulation of CSF System}

To simulate the CSF system in vitro, 3 containers were serially connected via flexible fluidic tubing. The main container, simulating the fluid compartment surrounding the brain (referred to as the brain reservoir), was initially filled with $200 \mathrm{ml}$ of saline ( $0.9 \%$ sodium chloride). This brain reservoir, representing the cranial cavity, was kept at a constant volume of $200 \mathrm{ml}$, and continuously stirred by a magnetic stirrer to achieve uniform distribution. A second reservoir, which is referred to as the source reservoir, was also filled with saline. This reservoir was connected to the brain reservoir container via soft tubing and a piezoelectric pump (Bartels Mikrotechnik). This reservoir acts to simulate the natural CSF creation of the ventricles. Saline was pumped from the source reservoir to the brain reservoir at a rate of $400 \mu \mathrm{l} / \mathrm{min}$; this rate was chosen to simulate the natural CSF creation rate of the ventricles.

A third container acted as the CSF sink, and is referred to as the sink reservoir. It was connected to the brain reservoir via additional soft tubing and provided a location for excess CSF to drain. Because the brain simulation container is airtight, its internal pressure increases when saline is pumped in from the source container, and it adjusts by compensatory outflow to the sink container. The sink reservoir thus simulates the absorption of CSF by the body. Altogether, the setup of this 3-reservoir fluidic circuit was intended to represent the fluid system surrounding the brain, inclusive of natural turnover of CSF within the CNS.

\section{Delivery of MTX to the In Vitro CSF System}

Lyophilized MTX sodium powder (Ben Venue Labs, Inc.) was reconstituted with $0.9 \%$ sodium chloride solution to $2.5 \mathrm{mg} / \mathrm{ml}$ and loaded into the MBP, which was connected to the brain container of the CSF in vitro system via 2 noncommunicating fluid tubes. The arrangement was bidirectional, with one tubing path for delivery of MTX and the other for sampling. The MTX was delivered to the brain reservoir according to the remotely programmed schedule, and it was quickly mixed using the magnetic stirrer. The presence of the simulated CSF source caused the MTX in the brain container to be gradually diluted and eliminated to the CSF sink, just as it would be in the human body. In parallel, samples from the brain container (500 $\mu$ l every $10 \mathrm{~min}$ ) were collected by the MBP, and the MTX concentration was determined by the onboard spectrophotometer. Absorbance was also measured using a Beckman DU730 spectrophotometer for comparison and validation.

\section{Fully Implantable Design}

The MBP is designed to be fully implantable and battery operated, much like a pacemaker, to lower the risk of infection and to allow the drugs to infuse over extended time periods. This improves patient quality of life by reducing time spent in the hospital and allowing more mobility and comfort. Self-sealing silicone septa allow access to the MBP medical reservoirs. An enclosure containing protruding ports can easily be felt through the skin for transcutaneous drug refill.

In patients, the future implantation technique for the MBP will be similar to a ventriculoperitoneal shunt. ${ }^{20}$ A right frontal curvilinear incision and bur hole will be made for the catheter placement, with a horizontal incision made approximately $2 \mathrm{~cm}$ superior to the nipple line as the pocket for the pump itself. A shunt passer will be tunneled from a postauricular nick to the chest incision. Two fer- 
rules in the pump are used to hook up the double-lumen catheter, which is moved superiorly through the shunt passer to the postauricular nick. A short shunt passer will be passed from the right frontal bur hole to the postauricular nick, and the catheter will be passed up to the frontal bur hole. The double-lumen ventricular catheter will be passed into the frontal horn of the lateral ventricle, and tied to a reservoir that connects the ventricular device with the one directed to the pump.

\section{Animal Experiments}

All animal protocols were approved by the Institutional Animal Care and Use Committee (IACUC) at CedarsSinai Hospital in Los Angeles, California. Four 15- to 18-week-old male swine were obtained from S\&S Farms. One animal per day underwent operation. All animals were anesthetized using propofol infusion, and maintained under anesthesia throughout the length of the procedure. After anesthesia, the pig was placed in a lateral position, and the lower back was prepared and shaved in a sterile fashion. The lumbar drain insertion site was localized using a lateral C-arm x-ray, approximately $2 \mathrm{~cm}$ superior to the iliac crest. A Codman lumbar drain was inserted using an 18-gauge Tuohy needle. For the ventriculostomy place- ment, a preoperative CT scan was obtained. First, by using the axial, sagittal, and coronal views of the CT scan, an entry site, proposed trajectory, and depth of catheter insertion were determined. At the frontal skull, a linear scalp incision at the insertion site was made and a hand-held perforator was used to make a frontal bur hole. The dura mater was opened with a pinpoint cautery, and a ventricular Codman catheter was inserted. Once CSF was verified, the catheter was tunneled and connected to the pump.

\section{Results \\ The MBP Setup}

The MBP is a fully implantable infusion pump with Wi-Fi biofeedback capabilities, designed to deliver chemotherapeutic agents locally and metronomically in a long-term therapy regimen. As outlined in Fig. 1, the MBP is fully implantable in the chest (above the pectoralis muscle similar to a pacemaker) or in the abdominal cavity (similar to a pain management infusion pump). The enclosure of the MBP is made of medical-grade, lightweight titanium alloy, which is widely used in many long-term implanted devices, due to the alloy's proven biocompatibility with tissue and bone. The pump is connected to a delivery

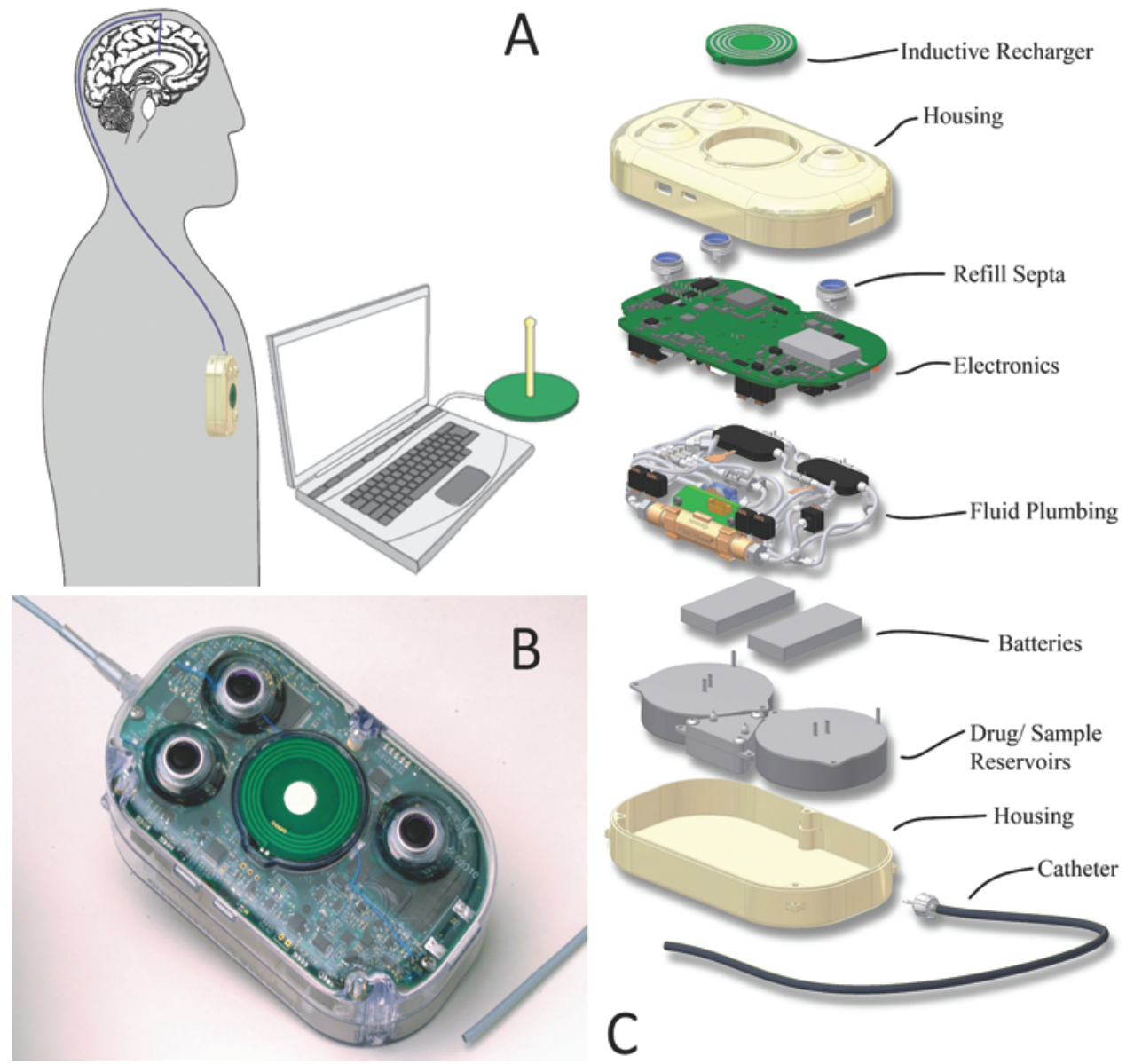

FIG. 1. The MBP components. A: The device can be implanted in the patient's chest or placed anywhere in the abdominopelvic cavity, with a catheter connecting to a ventricle in the brain. The wireless link allows clinicians to interact with the MBP via remote computer station. B: Exterior of the MBP (catheter only partially shown). C: Arrangement of individual parts configuring the MBP. Figure is available in color online only. 
target location, such as a tumor or a lateral ventricle, by a subcutaneous, dual-lumen catheter. Two micropumps inside the MBP, whose flow rates can be calibrated to approximately $500 \mu \mathrm{l} / \mathrm{min}$, independently control the flow of each lumen, which enables both the pumping of drug from its reservoir to the brain and simultaneous acquisition of CSF samples for analysis by the onboard sensor. Alternatively, both lumens can be used for the concurrent delivery of 2 different chemotherapeutic agents for combination therapy purposes. In the following material, we will primarily present characteristics of the bidirectional (simultaneous delivery and sampling) option.

The MBP is capable of interfacing to a computer base station near the patient. The sensor data are transmitted over the medical implant communication service wireless radio link to the care provider's computer for real-time monitoring, and this bidirectional link permits the user to immediately gain control of the implanted system and execute a new pumping routine. The intuitive computer interface enables clinicians to review current and past drug delivery and allows them to program individualized dosage adjustments over the course of chemotherapy.

To characterize MBP function, we designed a set of in vitro experiments to test the performance of its onboard spectrophotometer, its ability to perform metronomic deliveries, and its capacity to sample fluid and analyze it on board. Additional testing was performed, in which the pump was run in a closed-loop configuration, to show its ability to self-regulate the delivery of medication based on the drug concentration levels detected by the onboard sensor.

\section{Operational Evaluation of the MBP With In Vitro CSF System}

An in vitro CSF system was designed and set up as shown in Fig. 2 (and described in detail in the Methods section). The system was applied first to evaluate a comparison of metronomic versus bolus delivery of MTX over the course of 7 days. Metronomic delivery was programmed for 2 different doses given every 2 hours, with $0.298 \mathrm{mg}$ or $1.49 \mathrm{mg}$ injected in a volume of $0.416 \mathrm{ml}$ (resulting in a total dosage of $25 \mathrm{mg}$ or $125 \mathrm{mg}$, respectively, over 7 days). Bolus delivery consisted of a single dose of $12.5 \mathrm{mg}$ in $5 \mathrm{ml}$ every 3.5 days (resulting in $25 \mathrm{mg}$ total over 7 days). In all cases, the total volume injected over the course of 7 days was the same $(10 \mathrm{ml})$. The concentration of MTX achieved in the brain reservoir was measured by the MBP onboard spectrophotometer (Fig. 3).

As shown in Fig. 3, metronomic delivery of low-dose or high-dose MTX resulted in drug concentrations within the in vitro CSF that remained within a narrow concentration range, where highs and lows fluctuated by only about $20 \%$. In contrast, bolus injections resulted in extreme variations of CSF drug concentrations that ranged from a sharp spike immediately after delivery to undetectable drug levels within 48 hours. Altogether, these results demonstrate reliable metronomic delivery of MTX, which minimizes peak toxicity levels while increasing the duration of the therapeutic range.

To verify the accuracy of onboard MBP drug measurements, we compared them to a MATLAB simulation. In

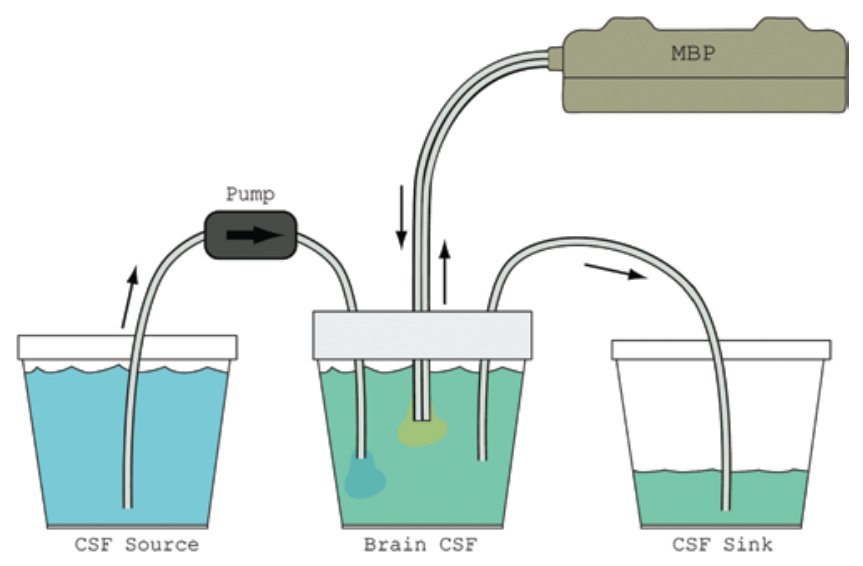

FIG. 2. Simulation of CSF system in vitro. A tightly sealed container (brain CSF, shown in the middle) is filled with $200 \mathrm{ml}$ saline to represent CSF in the cranial cavities. The MBP is connected via a dual-lumen catheter, which metronomically delivers drug and simultaneously collects CSF samples ( $500 \mu \mathrm{l} / \mathrm{sample})$ as per a preset schedule. The CSF source (left) contains $200 \mathrm{ml}$ saline and simulates natural CSF production by pumping liquid $(400 \mu \mathrm{l} / \mathrm{min})$ into the brain CSF (center) with the use of a piezoelectric pump. The CSF sink (right) acts as a sink and collects excess fluid draining from the brain CSF container, thereby simulating the absorption of CSF by the body. Uniform distribution of the delivered drug (i.e., MTX) was achieved by continuous mixing of the brain CSF with a magnetic stirrer. Due to the airtight seal of the brain CSF container, its volume remained constant at $200 \mathrm{ml}$ throughout the measurements. Figure is available in color online only.

this case, 12 doses ( $0.8 \mathrm{mg}$ in $0.32 \mathrm{ml}$ each) of MTX were delivered by MBP every 2 hours over a 24-hour period. In parallel, MATLAB (MathWorks) simulated delivery of 12 doses at $1.04 \mathrm{mg}$ in $0.416 \mathrm{ml}$ each. As shown in Fig. 4, in both cases the expected drug dosages could be confirmed, indicating a high degree of reliability of MBP drug delivery and subsequent measurements.

We also performed short-term measurements over the course of 30 minutes, with MTX injections every 10 minutes and real-time measurements of drug concentrations made by the onboard MBP ultraviolet photo sensor. We then compared the calculated expected drug concentrations to the actual, real-time concentrations of MTX. As shown in Fig. 5, there was close alignment between expected and actual drug concentrations, further verifying the high degree of reliability of MBP onboard measurements.

\section{Functional Evaluation of the MBP In Vivo}

The MBP was tested for reliability, consistency, and accuracy based on delivery of a contrast agent to a swine model in vivo. Additional objectives were to verify continuous CSF circulation from the ventricles to the lumbar thecal sac, to demonstrate the ability of the implanted MBP to continue its wireless communication while subcutaneously implanted, and to investigate how different modes of delivery would affect the concentration of the delivered agent within the cisterna magna. (See Methods section for surgical procedures and placing of the MBP.)

A total of 4 animals were studied to evaluate the pump and valve mechanisms for accuracy in pumping and ability to deliver contrast agent (methylene blue). In particular, we analyzed the MBP's flow rate and pump strength, and 


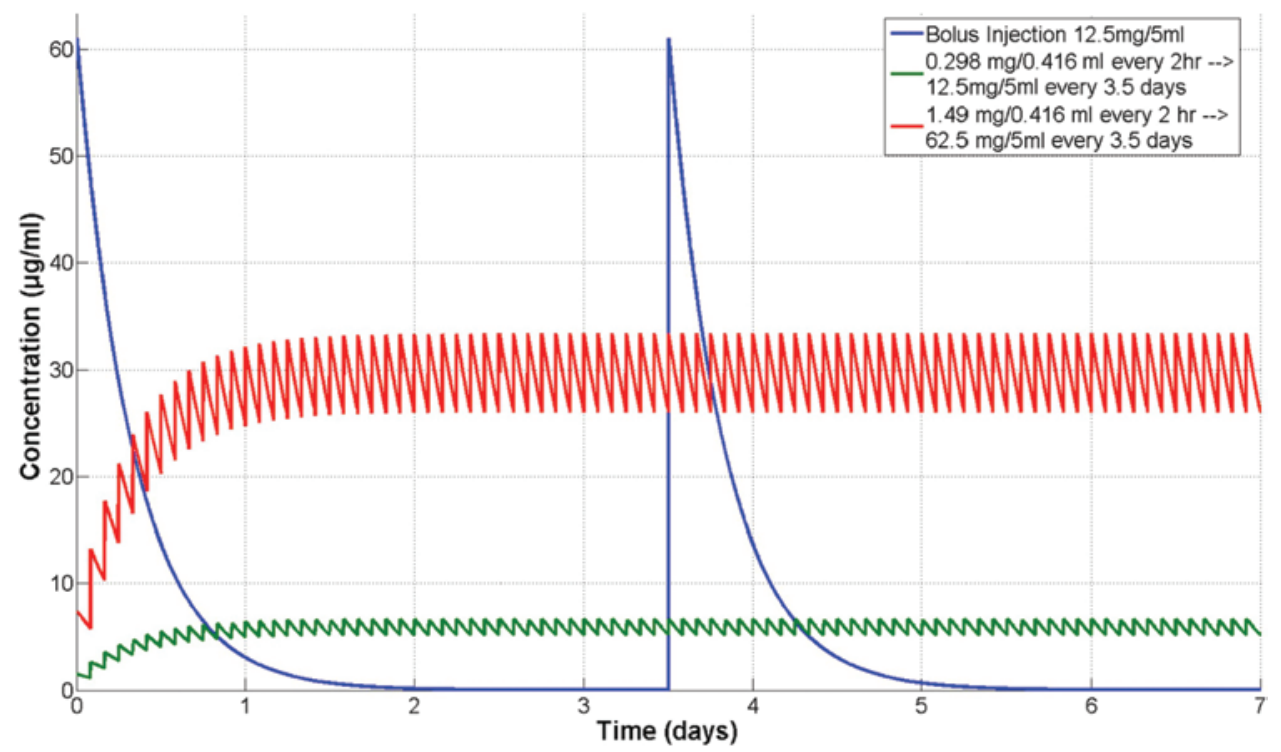

FIG. 3. Chart showing metronomic versus bolus delivery. Metronomic delivery consisted of 2 different doses $(0.298$ [green line] or 1.49 [red line] $\mathrm{mg}$ in a volume of $0.416 \mathrm{ml}$ ) administered every 2 hours over the course of 7 days, resulting in total dosages of 25 and $125 \mathrm{mg}$. The dose for bolus delivery was $12.5 \mathrm{mg}$ in a volume of $5 \mathrm{ml}$, administered at Time 0 and again after 3.5 days (blue line), resulting in a total dosage of $25 \mathrm{mg}$ over 7 days. Note that metronomic-based delivery minimizes peak levels, while increasing duration in the therapeutic range. Figure is available in color online only.

its ability to deliver and maintain a predetermined concentration of agent. The sensors were tested for accuracy in reading bellows volume, as well as internal and external pressure. At the end of the studies, a pathology procedure was conducted to investigate the concentration of contrast agents within the tissue, as well as the concentration of MTX in the brain. The latter agent was included with the objective being to illustrate that it was possible to measure MTX concentrations using a spectrophotometer (rather than for pharmacological performance). In addition, the MBP was examined for damage or corrosion, and for buildup of solids within the catheter and other CSFexposed components.

Figure 6 presents several MBP characteristics in vivo. Panel A of Fig. 6 shows the pump's increasing strength in relation to its flow over the course of 1 hour. While the

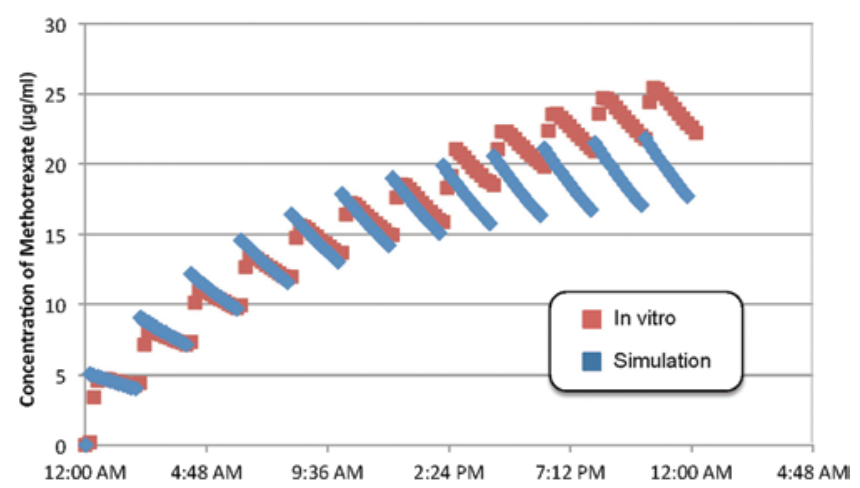

FIG. 4. Graph showing real-time measurements of MTX concentration change. Change of MTX concentration was detected by the MBP's spectrophotometer. Twelve doses were delivered over a 24-hour period (once every 2 hours). Each dose in the in vitro test contained $0.8 \mu \mathrm{g}$ MTX in a volume of $320 \mu$ l. Each dose in the MATLAB stimulation contained $1.04 \mu \mathrm{g}$ in $416 \mu \mathrm{l}$. Figure is available in color online only. flow rate hovers around the target pump rate, in this case $500 \mu \mathrm{l} / \mathrm{min}$, the bellows empty out, which generates a greater pressure differential for the pump to overcome. As a compensatory consequence, the pump strength increases for each subsequent pumping, thus in fact operating as intended. Panel B of Fig. 6 presents a comparison between expected and actually measured bellows volumes over the course of 1 hour and a total volume delivery of about 3 $\mathrm{ml}$. As shown, both levels are closely aligned. Panel C of Fig. 6 displays ICP readings over a duration of 7 hours, comparing readings from the output and inlet pressure sensors. This result indicates that, although both sensors

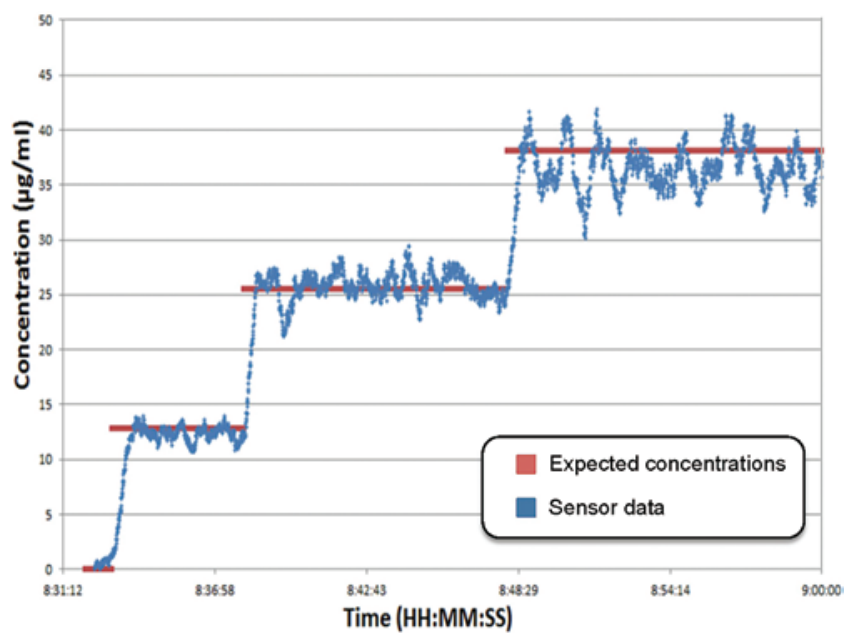

FIG. 5. Graph showing real-time measurement of MTX concentrations. The MTX was injected every 10 minutes over the course of 30 minutes, and measurements were performed in real time by the ultraviolet photo sensor at a wavelength of $302 \mathrm{~nm}$. The measured drug concentrations (blue line) were compared with the calculated expected drug concentrations (red lines). Figure is available in color online only. 

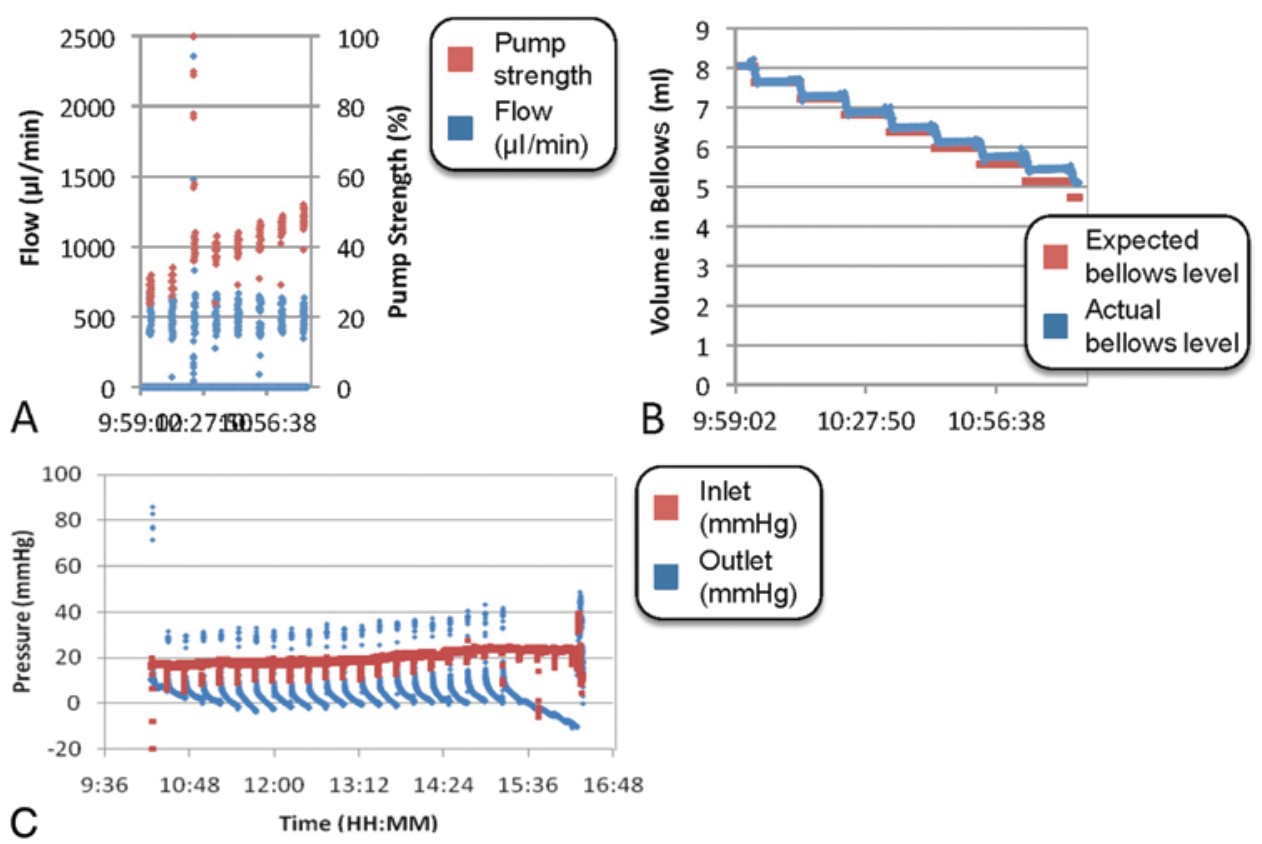

FIG. 6. Characteristics of the MBP in vivo. A: The flow rate (left vertical axis) was plotted over time (90 minutes) along with the pump's percent maximum strength (right vertical axis). B: The readings from pressure sensor 4 (output pressure sensor) are shown, where blue displays the continuous readings and red are the readings when the path between the CSF and sensor was open to obtain proper ICP readings. C: The readings from the output sensor and inlet pressure sensor are shown, where blue displays the outlet and red are readings from the inlet. Figure is available in color online only.

work reliably, the inlet pressure sensor appears to produce a more stable reading and is probably a better candidate for estimating ICP in the subject.

Figure 7 displays a comparison of the programmed target flow rate (for this test, $1250 \mu \mathrm{l} / \mathrm{min}$ ) with the actual real-time flow rate. As shown, the actual flow rate remains fairly constant over the 6-hour testing period, indicating reliable drug delivery in vivo.

Figure 8 displays a number of vital signs obtained from each pig. As shown, the majority of vital signs remained normal throughout the experimental period, indicating that placement and activation of the MBP was well tolerated by the animals. Of note, there was an increase in systolic blood pressure, which might have been second- ary to the transient increase in $\mathrm{ICP}-$ although there was no significant decrease in pulse, consistent with Cushing's mechanism..$^{15}$

At the end of the experiment, the pigs' brains and spinal cords were harvested and analyzed for the distribution of methylene blue and MTX concentration. In the case of methylene blue, the spectrophotometer was able accurately to measure the concentration of this dye achieved in vivo (Fig. 9A). Furthermore, MBP-mediated delivery of methylene blue to pig brain resulted in diffusion of dye through the entire ventricular system; dye was detectable in brainstem, cerebellum, and spinal cord (Fig. 9B and C). During MBP-mediated MTX delivery, MTX concentrations were measured in CSF samples obtained by lumbar drain (i.e.,

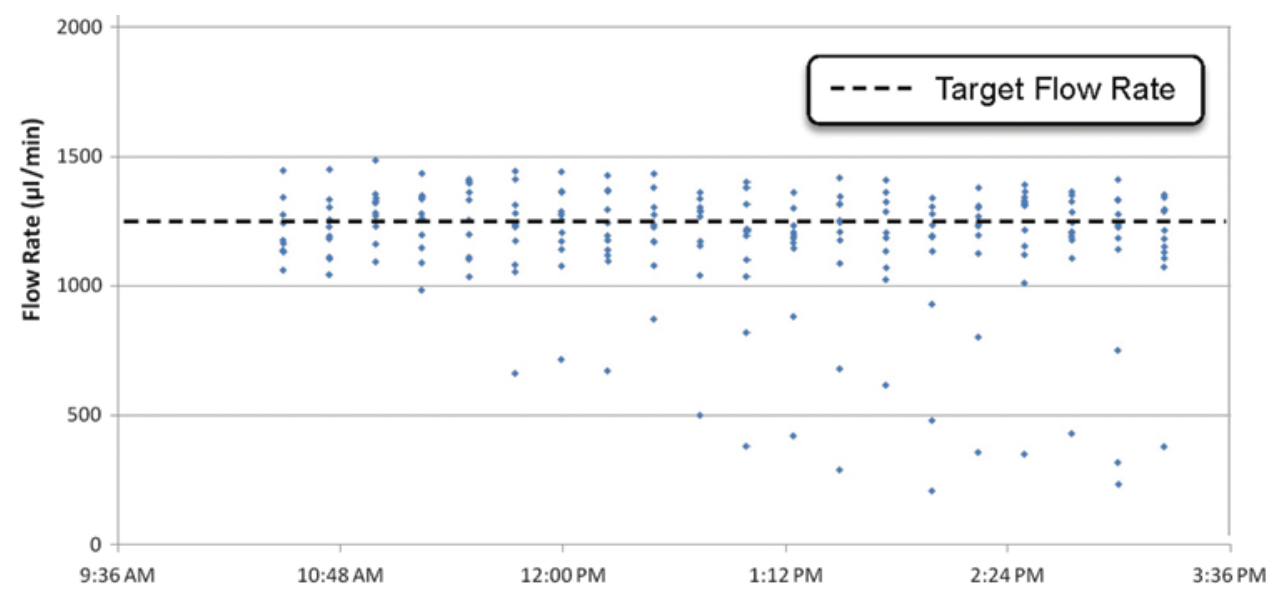

FIG. 7. Plot showing the flow rate. The programmed target flow rate $(1250 \mu \mathrm{l} / \mathrm{min})$ was compared with the actual, real-time flow rate over the course of 6 hours. Measurements were taken every 15 minutes. Figure is available in color online only. 


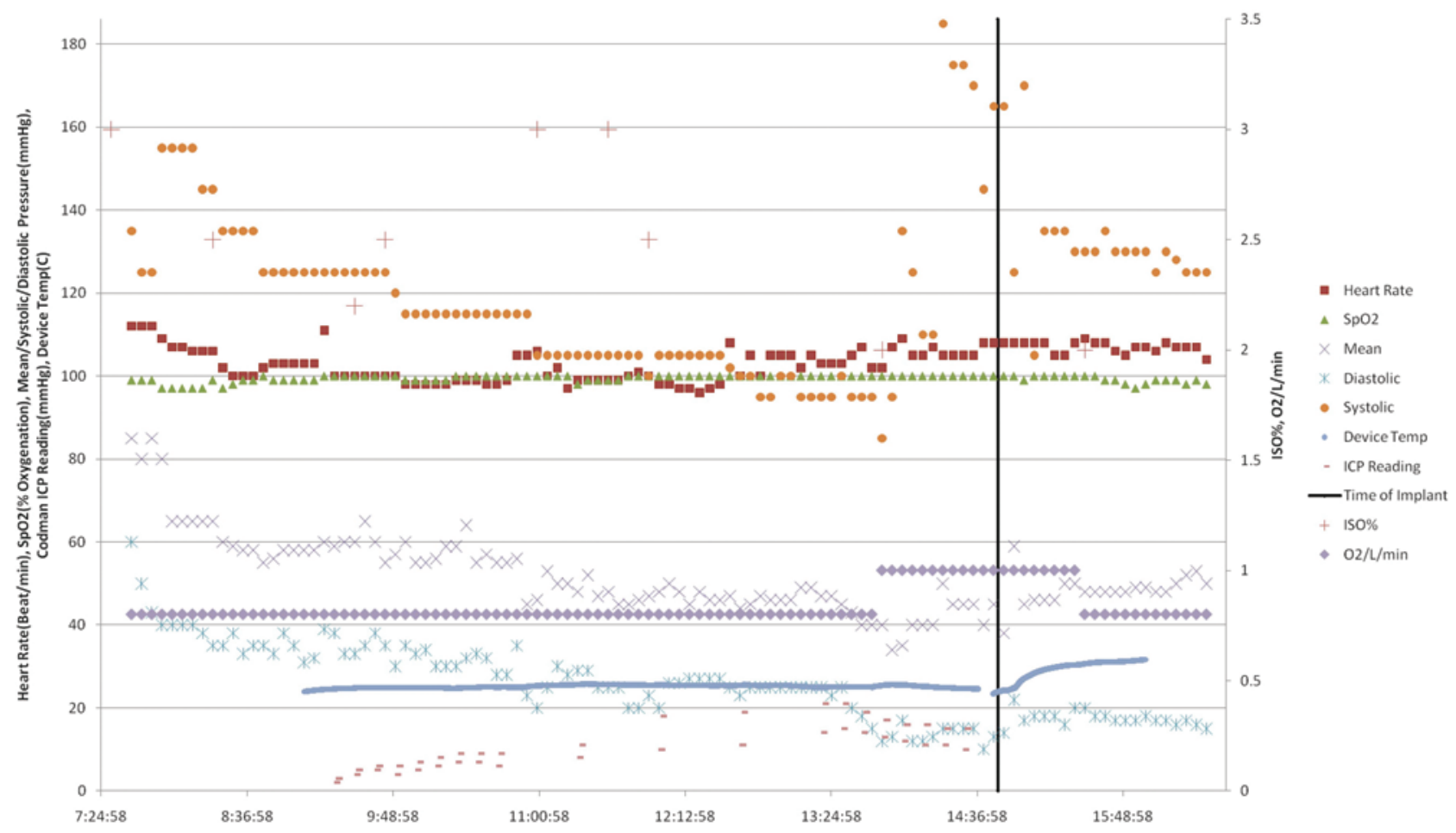

FIG. 8. Plot showing vital signs. Vital signs and other parameters were monitored before and after implantation of the MBP. The following parameters were included: heart rate, blood pressure (systolic and diastolic), blood oxygen saturation level $\left(\mathrm{SpO}_{2}\right), 0 x y-$ gen consumption $\left(\mathrm{O}_{2} / \mathrm{L} / \mathrm{min}\right)$, and ICP. ISO $\%$ = vaporizer percentage of isoflurane. Figure is available in color online only.

distant from the catheter delivery site) over the course of several hours. As shown in Fig. 9D, high concentrations of the drug could be documented in the CSF at levels that compared favorably to what has been reported in patients (e.g., Strother et al. ${ }^{22}$ ). After initial buildup, MTX concentrations remained fairly constant for several hours, consistent with metronomic delivery. On termination of drug delivery, MTX concentrations began to decline rapidly.

Together, these results demonstrate that the device and all of its sensors function as expected in vivo, and are able to deliver and measure the programmed dosages of pharmacological agents accurately and effectively. The findings further demonstrate that CSF could readily be accessed from the ventricles via the double-lumen catheter, that wireless communication with the implanted MBP can easily be established, and that no significant physiological changes in blood pressure, pulse, or ICP occurred during infusion into the CSF of the animals.

\section{Discussion}

\section{Current Limitations in LC Treatment}

Treatment outcome for LC has not significantly improved over the past 10 years, and the dismal prognosis invariably remains. ${ }^{1,3}$ Traditionally, chemotherapeutic treatment for LC has consisted of MTX or Ara-C, and in recent years the use of a liposomal formulation of Ara-C (DepoCyt) has been favored.${ }^{14}$ Newer approaches to LC treatment involve biological agents, such as trastuzumab (Herceptin; Genentech, Inc.) for Her2/neu-positive breast cancer LC, or rituximab (Rituxan, Biogen Idec and Genentech, Inc.) for lymphomatous carcinomatosis, which have demon- strated efficacy in small clinical trials and case reports..$^{18}$ These monoclonal antibodies are unable to cross the BBB and therefore require direct intrathecal administration, similar to MTX and DepoCyt. More recent developments involve combination approaches with the aforementioned agents. ${ }^{10,13}$ Although some encouraging outcomes have been noted, the median survival is still far from satisfactory, and substantial additional improvements in LC therapy are required.

\section{Use of the MBP for Treatment of LC}

The MBP was designed to address the most pressing problems and limitations in treating patients with LC. Its key advantages over current treatments include the following. 1) The application of biofeedback will measure and report intrathecal drug concentrations, which will enable the clinician to promptly adjust drug dosages. 2) The rate of drug delivery can be adjusted without the need for injections, which greatly reduces the risk of infections and enables fine-tuned adjustments to maintain effective intrathecal drug concentrations. 3) The digital interface can be programmed so that predetermined drug dosages and delivery rates are adjusted automatically to balance potential fluctuations of in situ drug concentrations. 4) The option to accommodate several bellows enables delivery of various therapeutic agents from a single device, which obviates the need for multiple, repeated injections in case of combination therapy. 5) Metronomic delivery (i.e., frequent small drug doses over a short interval) is expected to reduce the incidence of complications, because it enables long-term CSF exposure to drug concentrations within the therapeu- 

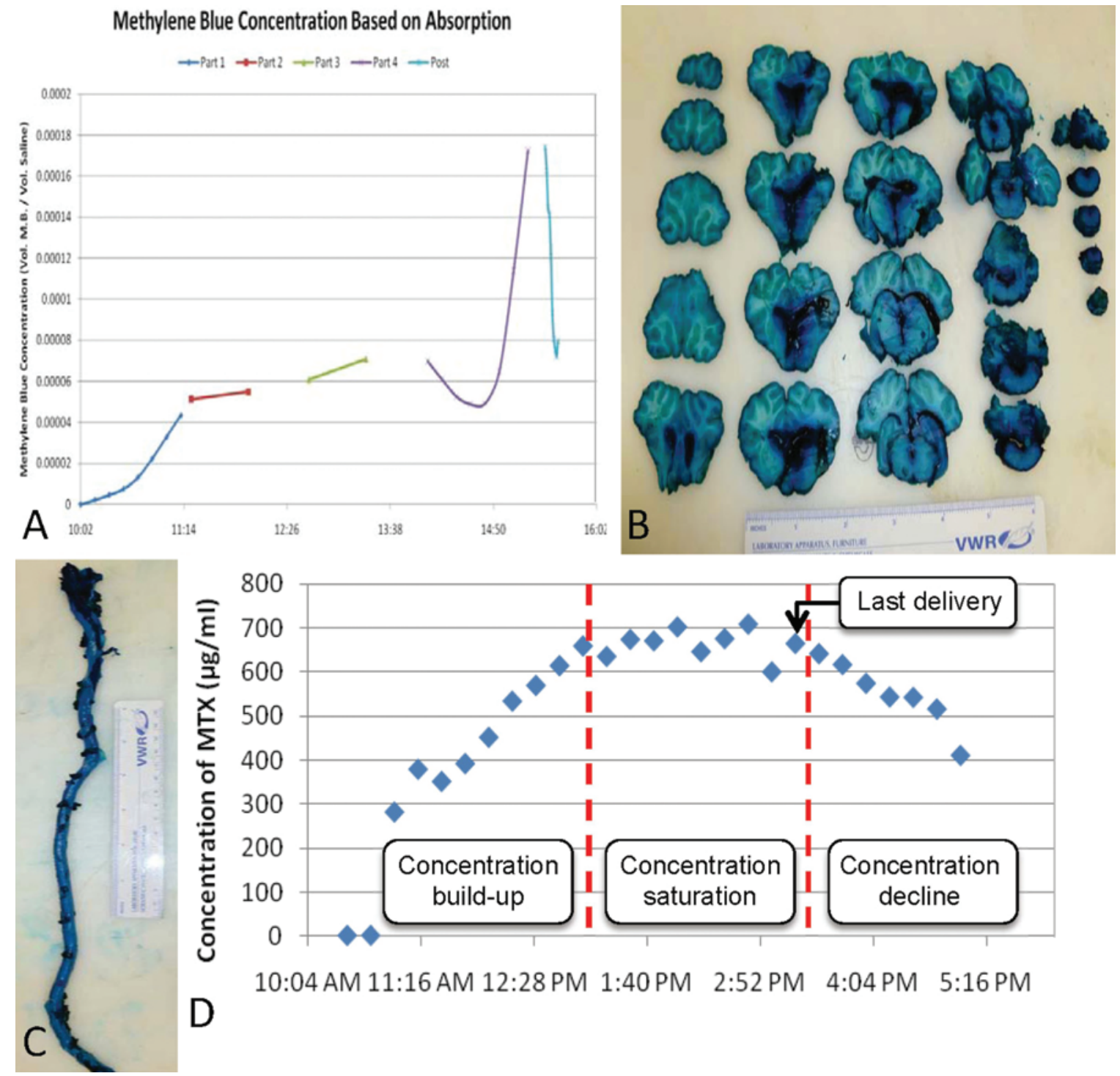

10:04 AM 11:16 AM 12:28PM 1:40PM 2:52 PM 4:04PM 5:16PM

FIG. 9. Drug distribution in pig CNS. A: Graph showing the concentrations of methylene blue, plotted on a concentration versus time curve. B and C: Photographs of dissected brainstem, cerebellum, and spinal cord, showing diffusion of the dye through the entire ventricular system of the pigs. (Ruler increments closest to the specimens are in inches.) D: Chart showing concentrations of MTX in CSF samples obtained by lumbar puncture. Injection of MTX via MBP was terminated at 3:20 PM, after which time the drug concentration began to decline. Figure is available in color online only.

tic range, while avoiding excessively high and potentially neurotoxic drug levels. 6) Using a stock concentration of $25 \mathrm{mg} / \mathrm{ml}$ MTX in a 5-ml reservoir, the MBP can be used to deliver the metronomic drug regimen for 6 months without refill, and thus reduce the number of skin piercings and injections of conventional treatments. Altogether, therefore, clinical application of the MBP is expected to maximize therapeutic efficacy while minimizing toxicity; it therefore has the potential to prolong patient survival along with improved quality of life.

\section{Potential Limitations of MBP Use}

Leptomeningeal carcinomatosis is not a disease in which there is uniform spread of cancer cells throughout the CSF, and therefore local tumor deposits can cause poor circulation of a drug throughout the CNS axis. As a re- sult, drug circulation may be limited in some regions of the CNS where there is such occlusion. While this problem also impairs conventional intrathecal chemotherapy via Ommaya reservoir or lumbar puncture, it may exert limitations on therapy with the MBP as well.

Another complicating factor in LC is hydrocephalus, which is seen in approximately $25 \%-40 \%$ of these patients, and currently is addressed by placing a shunt that can be adjusted at the time of intrathecal chemotherapy delivery. ${ }^{3,11}$ The MBP can be adapted for patients with hydrocephalus. Future designs will have a catheter extending from the collection bellows that can be inserted into the peritoneum.

Further problems may be posed by catheter occlusion caused by the high protein concentration and cancer cells present in the CSF. The delivery of drugs into the CSF via 
the outflow ventricular catheter (which should theoretically be less prone to occlusion than a shunt catheter, based on the chronic positive pressure and outflow) is worth investigating. In comparison, the inflow catheter, where CSF is actively sucked into the MBP via negative pressure, may have a higher risk of occlusion. However, this inflow lumen features a port where external saline can be injected to unplug the catheter, thereby greatly reducing the risk of catheter occlusion.

Potential pump failure may result from malfunctions in the communication, fluid path, and control components, or the catheter. A communication malfunction may result from a number of causes, such as electromagnetic interference, failure of the wireless Internet connection, disconnected antenna, or the device being out of range, which could cause cessation of communication or reduced signal quality with intermittent communication. Clogged or leaky fluid paths, possibly due to a residue deposit, a kink, a loose connection, or a break in the tubing, may cause the pump to stall or the battery to drain faster due to overcompensation. Although such occurrences may not be entirely avoidable, their risks for the patient will be greatly reduced by having leakage, pressure, and current sensors in the MBP that will alert the physician to the existence of such problems.

Metronomic chemotherapy has been proposed as a means to potentiate the antitumor effects of chemotherapeutic drugs and to overcome drug resistance,, 19 and the MBP presents a technical device aimed at achieving this goal. Although the literature provides supportive evidence that metronomic approaches indeed might be superior to bolus delivery, ${ }^{9,19}$ definitive proof for this claim has not yet been established. Therefore, future investigations using the MBP will have to include studies to evaluate the pump's therapeutic efficacy in comparison with conventional bolus delivery of chemotherapeutic drugs.

\section{Other Applications of the MBP}

The MBP is currently designed for chemotherapeutic drug delivery into the CSF in cases of LC. However, additional future clinical applications for the brain are anticipated. Drug delivery into the CSF for the treatment of primary brain cancers such as malignant gliomas or metastatic cancers is an obvious option, because it would overcome the BBB, which represents a serious obstacle for many chemotherapeutic drugs that are delivered via intravenous or oral administration. Future potential CNS applications include direct CSF delivery of drugs for refractory fungal meningitis (e.g., coccidiomycosis, cryptococcosis), which currently is treated with Ommaya reservoir-based delivery of amphotericin; other applications may include Parkinson disease, Alzheimer disease, stroke, and other neurodegenerative diseases of the brain.

\section{Future Studies and Regulatory Approval}

Future studies will extend the in vivo Phase I nonsurvival studies described in this current report to in vivo Phase II survival studies in a pig model, and will include analysis of long-term toxicity. The data from these future studies will be used for an ISO 13485 application and the design of good laboratory practice for large-animal stud- ies. Investigational device exemption approval will then be obtained for Phase I trials in the US and Europe for patients with LC. These initial trials will be using a concentration $\times$ time regimen for MTX and will apply accepted regimens for that agent (currently delivered via Ommaya reservoir). The goal will be to determine the accuracy and safety of the pump, and eventually to enable customized delivery of MTX via in vivo verification of real-time drug concentrations.

\section{Acknowledgment}

We acknowledge support by the engineering and office staff of the Pharmaco-Kinesis Corporation.

\section{References}

1. Cavaliere R, Schiff D: Chemotherapy and cerebral metastases: misperception or reality? Neurosurg Focus 22(3):E6, 2007

2. Chamberlain MC, Kormanik PA, Barba D: Complications associated with intraventricular chemotherapy in patients with leptomeningeal metastases. J Neurosurg 87:694-699, 1997

3. Clarke JL: Leptomeningeal metastasis from systemic cancer. Continuum (Minneap Minn) 18:328-342, 2012

4. Drappatz J, Batchelor TT: Leptomeningeal neoplasms. Curr Treat Options Neurol 9:283-293, 2007

5. Fleischhack G, Jaehde U, Bode U: Pharmacokinetics following intraventricular administration of chemotherapy in patients with neoplastic meningitis. Clin Pharmacokinet 44:1-31, 2005

6. Gabay MP, Thakkar JP, Stachnik JM, Woelich SK, Villano JL: Intra-CSF administration of chemotherapy medications. Cancer Chemother Pharmacol 70:1-15, 2012

7. Glantz MJ, Hall WA, Cole BF, Chozick BS, Shannon CM, Wahlberg L, et al: Diagnosis, management, and survival of patients with leptomeningeal cancer based on cerebrospinal fluid-flow status. Cancer 75:2919-2931, 1995

8. Glantz MJ, Jaeckle KA, Chamberlain MC, Phuphanich S, Recht L, Swinnen LJ, et al: A randomized controlled trial comparing intrathecal sustained-release cytarabine (DepoCyt) to intrathecal methotrexate in patients with neoplastic meningitis from solid tumors. Clin Cancer Res 5:33943402, 1999

9. Kerbel RS, Kamen BA: The anti-angiogenic basis of metronomic chemotherapy. Nat Rev Cancer 4:423-436, 2004

10. Kim DY, Lee KW, Yun T, Park SR, Jung JY, Kim DW, et al: Comparison of intrathecal chemotherapy for leptomeningeal carcinomatosis of a solid tumor: methotrexate alone versus methotrexate in combination with cytosine arabinoside and hydrocortisone. Jpn J Clin Oncol 33:608-612, 2003

11. Lin N, Dunn IF, Glantz M, Allison DL, Jensen R, Johnson $\mathrm{MD}$, et al: Benefit of ventriculoperitoneal cerebrospinal fluid shunting and intrathecal chemotherapy in neoplastic meningitis: a retrospective, case-controlled study. J Neurosurg 115:730-736, 2011

12. Mechleb B, Khater F, Eid A, David G, Moorman JP: Late onset Ommaya reservoir infection due to Staphylococcus aureus: case report and review of Ommaya infections. J Infect 46:196-198, 2003

13. Mego M, Sycova-Mila Z, Obertova J, Rajec J, Liskova S, Palacka P, et al: Intrathecal administration of trastuzumab with cytarabine and methotrexate in breast cancer patients with leptomeningeal carcinomatosis. Breast 20:478-480, 2011

14. Murry DJ, Blaney SM: Clinical pharmacology of encapsulated sustained-release cytarabine. Ann Pharmacother 34:1173-1178, 2000 
15. Paton JF, Dickinson CJ, Mitchell G: Harvey Cushing and the regulation of blood pressure in giraffe, rat and man: introducing 'Cushing's mechanism.' Exp Physiol 94:11-17, 2009

16. Pavlidis N: The diagnostic and therapeutic management of leptomeningeal carcinomatosis. Ann Oncol 15 (Suppl 4):iv285-iv291, 2004

17. Pentheroudakis G, Pavlidis N: Management of leptomeningeal malignancy. Expert Opin Pharmacother 6:1115-1125, 2005

18. Perissinotti AJ, Reeves DJ: Role of intrathecal rituximab and trastuzumab in the management of leptomeningeal carcinomatosis. Ann Pharmacother 44:1633-1640, 2010

19. Pietras K, Hanahan D: A multitargeted, metronomic, and maximum-tolerated dose "chemo-switch" regimen is antiangiogenic, producing objective responses and survival benefit in a mouse model of cancer. J Clin Oncol 23:939-952, 2005

20. Raysi Dehcordi S, De Tommasi C, Ricci A, Marzi S, Ruscitti $\mathrm{C}$, Amicucci G, et al: Laparoscopy-assisted ventriculoperitoneal shunt surgery: personal experience and review of the literature. Neurosurg Rev 34:363-371, 2011

21. Sandberg DI, Bilsky MH, Souweidane MM, Bzdil J, Gutin
PH: Ommaya reservoirs for the treatment of leptomeningeal metastases. Neurosurgery 47:49-55, 2000

22. Strother DR, Glynn-Barnhart A, Kovnar E, Gregory RE, Murphy SB: Variability in the disposition of intraventricular methotrexate: a proposal for rational dosing. J Clin Oncol 7:1741-1747, 1989

\section{Author Contributions}

Conception and design: Chen, Shachar. Acquisition of data: Chen, Napolitano, Adell. Analysis and interpretation of data: Chen, Schönthal, Shachar. Drafting the article: Chen, Schönthal, Shachar. Critically revising the article: Chen, Schönthal, Shachar. Reviewed submitted version of manuscript: all authors. Administrative/technical/material support: Napolitano, Adell. Study supervision: Chen, Shachar.

\section{Correspondence}

Thomas C. Chen, Department of Neurosurgery, University of Southern California, 2011 Zonal Ave., Los Angeles, CA 90089. email: thomas.chen@med.usc.edu. 